\title{
HIV INCIDENCE AMONG MEN WHO HAVE SEX WITH MEN AT A COMMUNITY-BASED FACILITY IN GREECE
}

\author{
Georgios K. Nikolopoulos ${ }^{1}$, Sophocles Chanos², Emmanouil Tsioptsias ${ }^{2}$, loannis Hodges-Mameletzis ${ }^{3}$, Dimitra \\ Paraskeva ${ }^{4}$, Nikos Dedes ${ }^{2}$ \\ ${ }^{1}$ Medical School, University of Cyprus, Nicosia, Cyprus \\ ${ }^{2}$ Ath Checkpoint, Athens, Greece \\ ${ }^{3}$ World Health Organization, Geneva, Switzerland \\ ${ }^{4}$ Hellenic Centre for Disease Control and Prevention, Amarousio, Greece
}

\begin{abstract}
SUMMARY
Objective: Men who have sex with men (MSM) are disproportionately affected by HIV in Greece. However, research on HIV incidence in this group is lacking. This study aimed at estimating HIV incidence among MSM in Athens, Greece.

Methods: The analysis included routinely collected data between January 2013-June 2015 from adult MSM who visited a community-based facility (Ath Checkpoint) at least twice and were non-reactive to the rapid INSTIT HIV-1/HIV-2 assay at baseline. HIV conversion rates were calculated by dividing the number of clients who became reactive by the person-years of observation. All statistical analyses including Poisson regression models were conducted in STATA 14.

Results: A total of 1,243 MSM contributed 1,102.50 person-years (py). The overall (per 100 py) conversion rate was 3.99 (95\% Cl: 2.97-5.36). In multivariable analyses, age less than 30 years was associated with an increased risk of HIV conversion (rate ratio: $2.01 ; 95 \%$ Cl: 1.08-3.76).

Conclusions: This analysis shows high rates of HIV conversion among MSM who repeatedly visit a community-based testing site. Ath Checkpoint could contribute to HIV surveillance and identify a high-risk group that could benefit from essential health interventions.
\end{abstract}

Key words: HIV incidence, testing, MSM, checkpoint, intervention

Address for correspondence: G. K. Nikolopoulos, Medical School, University of Cyprus, Palaios Dromos Lefkosias-Lemesou 215/6 2029 Strovolos, Nicosia, Cyprus. E-mail: gknikolopoulos@gmail.com

https://doi.org/10.21101/cejph.a4856

\section{INTRODUCTION}

Globally, the HIV epidemic among men who have sex with men (MSM) shows no signs of recession $(1,2)$. The European Centre for Disease Control (ECDC) estimated that $42 \%$ of the HIV diagnoses in 2014 in the European Union and the European Economic Area (EU/EEA) occurred among MSM (3). Longitudinal studies on MSM in various settings indicated that HIV incidence ranges between $0.5 \%$ and $10 \%$ (1).

HIV in MSM remains a public health concern in Greece. Approximately half of the reported infections to the Greek Centre for Disease Control and Prevention are associated with male-to-male sexual transmission (4). The concentrated HIV epidemic in Greece over the years saw a shift in 2011 when an HIV outbreak in people who inject drugs (PWID) resulted in large numbers of HIV diagnoses between 2011 and 2013. Case reports in 2014-2015 show that the epidemic in PWID has been substantially contained while the majority of HIV infections are now diagnosed again among $\operatorname{MSM}(4,5)$.

HIV surveillance in Greece has been based on the mandatory reporting of HIV diagnoses from medical clinics and laboratories (6). The system is comprehensive with timely and accurate data collection. However, the epidemiological puzzle remains incomplete because Greece lacks input from second generation surveillance sources (6). Consequently, there is a paucity of critical information on prevalence, incidence, and behavioural patterns, particularly for key populations such as MSM. More recently, however, community-based and MSM-friendly testing facilities have been operating in urban settings and aim at filling essential gaps in knowledge and strategic information essential for public health planning, including epidemiological dynamics. Notably, the non-governmental organization (NGO) Positive Voice started an ambitious project in 2012 by instituting and operating a nonclinical HIV testing centre (Ath Checkpoint) in Athens.

Given the lack of reliable, real-time data on HIV transmission dynamics, coupled with the complex Greek environment characterized by social instability, economic constraints, and the aftermath of the recent outbreak among PWID, this study estimates and evaluates recent HIV incidence rates among MSM who repeatedly visited Ath Checkpoint for HIV testing.

\section{MATERIALS AND METHODS}

Ath Checkpoint is a community-based, rapid HIV testing and counselling site in downtown Athens, Greece*. Funded by the AIDS Healthcare Foundation (Los Angeles, USA), this free testing

*http://athcheckpoint.gr/english/ 
service has been operated by the NGO Positive Voice since November 2012. Ath Checkpoint also maintains collaborative links to other actors, including the Greek Liver Patients Association and the Municipality of Athens. The staff consists of experienced professionals and volunteers who have been trained by the Greek Centre for Disease Control and Prevention. The facility is centrally located, easily accessed using public transportation, and open from Monday to Saturday (12 noon -8 p.m.). Ath Checkpoint works typically with appointments but also addresses unscheduled visits. Although the primary target population are MSM, Ath Checkpoint offers free HIV testing and counselling to the entire population.

People who visit Ath Checkpoint, following a process of written and verbal information provided by the staff, are asked to orally and voluntarily consent to the registration and testing procedures of the facility. Verbal informed consent was chosen in order to increase testing rates by minimizing fears about data protection and limiting bureaucratic constraints, and data suggest that it is an adequate substitute for written consent (7). Clients provide, for purposes of data recording, a pseudonym and, subsequently, get pre-test counselling. An identification number is assigned to every client in order to match him/her with the consultation and the test result. A client who does not recall the first identification number or the pseudonym at his/her subsequent visits may get a new identifier. A short questionnaire collects information on gender, age, sexual orientation (MSM, heterosexuals, other), place of residence, and HIV testing history (number of previous tests).

HIV testing on fingerstick blood is performed using the IN$\mathrm{STI}^{\mathrm{TM}} \mathrm{HIV}-1 / \mathrm{HIV}-2$ assay and the result is available in 1 minute. When the test is reactive, the client is referred to hospital units specialized in HIV treatment and care for confirmatory testing, and if necessary, for clinical evaluation and psychological support. For non-reactive results at Ath Checkpoint, post-testing counselling is offered.

This analysis included data routinely collected from MSM aged $\geq 18$ years who visited Ath Checkpoint at least twice between January 2013 and June 2015, and were HIV negative at baseline. The matching of visits for the repeated testers was based on identification numbers, pseudonyms, age, place of residence, and number of visits. This work consists of secondary but necessary evaluation analyses of effectiveness in detecting undiagnosed infections (8) using limited anonymized data and therefore has not undergone through a regular process for ethical approval by a relevant committee. All clients verbally consented to using anonymized data for statistical analyses.

The rate of HIV conversion was calculated by dividing the number of clients who became reactive to the test by the person-years (py) of observation. Conversion was assumed to occur in the mid-point between the non-reactive and reactive test. Consequently, py for MSM who became reactive included half the time period from the last negative to the first reactive result. Descriptive statistics included frequencies/percentages for categorical variables and median/25th-75th percentiles for continuous parameters. The non-parametric Wilcoxon rank-sum test was used to test the hypothesis that two samples were drawn from the same distribution. The multivariable modelling included Poisson regression. Age was modelled both as a continuous and as a categorical variable. MSM were classified as older or younger than 30 years based on their median age value but also on the increased diagnosis rates that have been observed among MSM under 30 years of age (9). All statistical analyses were performed in STATA 14.

This analysis follows the guidelines (STROBE) for reporting observational studies (10).

\section{RESULTS}

In total, 1,254 MSM were initially non-reactive to the rapid HIV test and were examined at least twice between January 2013 and June 2015. Of these, 55 became reactive. Eleven subjects with reactive results but follow-up times less than three months were excluded from further analyses as they could have been in the window period. Therefore, the final analysis included 1,243 $\operatorname{MSM}(1,102.50$ person-years of observation) with a median age of 29 years (25th-75th percentile: $24-35)$, a median number of two visits (25th-75th percentile: $2-3$ ), and a median follow-up of 9.69 months (25th-75th percentile: $5.45-14.49)$. Age $(p=0.14)$ and number of visits $(p=0.85)$ were not significantly different between those who converted and those who remained non-reactive over the period of observation. In the final dataset, convertors had half the follow-up time of the non-reactive group because conversion was assumed to happen in the mid-point between the negative and reactive result. Otherwise, the follow-up times were similar $(\mathrm{p}=0.94)$.

The overall rate of conversion was 3.99 per 100 py with a $95 \%$ confidence interval (CI) of 2.97-5.36. As shown in Table 1, the rate was higher in those aged between 18 and 30 years, especially in the 25-30 years group (5.97 per 100 py; 95\% CI: $3.46-10.27$ ). The conversion rates showed a decreasing trend from 2013 (4.67 per 100 py; $95 \%$ CI: $2.71-8.05$ ) to 2014 (3.92 per 100 py; $95 \%$ CI: 2.65-5.79) and to the first half of 2015 (3.23 per 100 py; $95 \%$ CI: 1.45-7.19).

The three available variables (age, number of visits, calendar year) were simultaneously modelled using Poisson regression.

Table 1. HIV conversion rates (reactive to a rapid test following a baseline non-reactive result) by age group among 1,243 men who had sex with men and visited Ath Checkpoint, Athens, Greece, multiple times between January 2013 and June 2015

\begin{tabular}{|l|c|c|c|c|c|}
\hline Age group in years & Conversions & Person-years & Rate & $95 \%$ low limit & 95\% upper limit \\
\hline $18-25$ & 16 & 318.70 & 5.02 & 3.08 & 8.20 \\
\hline $25-30$ & 13 & 217.93 & 5.97 & 3.46 & 10.27 \\
\hline $30-40$ & 14 & 412.81 & 3.39 & 2.01 & 5.73 \\
\hline $40-50$ & 1 & 124.41 & 0.80 & 0.11 & 5.71 \\
\hline$\geq 50$ & 0 & 28.66 & 0 & - & - \\
\hline
\end{tabular}


Age was inserted either as a continuous variable or as a dichotomous factor (older or younger than the age of 30 years); 645 $(51.89 \%)$ MSM fell in the $<30$ years group and $598(48.11 \%)$ in the $\geq 30$ years category. The calendar year and the number of visits were not significantly associated $(p>0.05)$ with the natural logarithm of the conversion rate. On the other hand, the conversion rate decreases by $4 \%$ as a MSM becomes one year older (rate ratio per year increase in age adjusted for calendar year and number of visits: 0.96 ; 95\% CI: $0.92-0.99 ; \mathrm{p}=0.04$ ). The rate of conversion was twice as high for MSM younger than 30 years as for those older than 30 years $(p=0.03$; rate ratio for age adjusted for calendar year and number of visits: $2.01 ; 95 \%$ CI: $1.08-3.76)$.

\section{DISCUSSION}

Community-based approaches to HIV testing and counselling are demonstrating how key populations such as MSM can benefit from testing uptake and linkages to HIV treatment and care. Ath Checkpoint is a community-based facility that offers free rapid HIV testing and counselling services in Athens, Greece. Our study estimated a 4\% annual rate of HIV conversion in 1,243 MSM who repeatedly visited Ath Checkpoint between January 2013 and June 2015 . The rate was higher among persons younger than 30 years.

HIV continues to be increasing among MSM worldwide (2, 3). High rates of HIV incidence have been reported by communitybased testing services in Spain and Portugal $(11,12)$. There are additional challenges in Greece given the unstable socioeconomic background and the potential mid- and long-term consequences of the recent HIV outbreak among PWID $(4,6)$. Heightened rates of sexual transmission are likely to follow the HIV spread among drug injectors (4). In addition, bio-behavioural studies in MSM are discouragingly absent. The most recent estimate of HIV prevalence among MSM who got tested in Greece was around 13\% and was derived from self-reports in an internet-based study (13). The need to enhance HIV surveillance, especially for MSM, is urgent and a public health priority. The operation of community-based testing sites that target key populations could be useful as such sites can inform surveillance while fulfilling their primary aims of HIV testing, linkage to care, and fighting stigma.

HIV prevention for MSM requires an integrated and holistic approach that takes into account the biological, behavioural, network, and structural factors that enhance the risk for HIV acquisition in MSM. Promising treatment-based prevention interventions, i.e., treatment as prevention (TasP) and pre-exposure prophylaxis (PrEP), are critical elements of HIV combination prevention, and will likely enhance the essential strategies based on health education, condom use, and HIV testing and counselling (14). The World Health Organization has already issued guidelines (15) that recommend the use of PrEP in persons at substantial risk of HIV infection (with substantial risk defined with an incidence $>3 \%$ ). On the basis of the findings of our analysis, Ath Checkpoint could be the type of vehicle by which PrEP, condom distribution, and other interventions can be delivered effectively in Greece given that HIV incidence in MSM exceeds the WHO's threshold on substantial risk.

*https://eurohivedat.eu/
The results of our analysis should be interpreted carefully in light of some limitations. The primary operation of Ath Checkpoint is to provide testing services, although the research capacity is being strengthened (Euro HIV EDAT project)*. This study is not a classic cohort with scheduled follow-ups and regular collection of key indicators and detailed information. Consequently, unmeasured biases may have affected the results. In addition, it is particularly difficult to assess whether the sample of MSM who visit a non-clinical testing site is representative of the broader MSM population in Greece. Therefore, actual incidence rates could be lower than what was observed in our analysis. Nonetheless, our study provides important insight on how to identify MSM who face a considerable risk of HIV acquisition and who should be prioritized for prevention services to reduce risk of HIV infection.

As real full names are not documented and recorded at Ath Checkpoint, false matching of visits is likely. The matching procedure was challenging based on pseudonyms, identification numbers, age, place of residence, and numbers of reported previous visits. In terms of the rapid test used in our study, both sensitivity and specificity, as reported by the manufacturer, are very high. Furthermore, HIV infection was Western-Blot confirmed in more than $99 \%$ of people with reactive results at Ath Checkpoint who were referred to a medical clinic.

\section{CONCLUSIONS}

HIV testing and counselling remains the cornerstone of efforts to contain HIV infection. In addition to their underlying goal of testing provision, MSM-friendly sites such Ath Checkpoint may facilitate the collection of incidence data and could help identify groups of MSM with very high risk of HIV infection who could benefit from essential interventions.

\section{Acknowledgements}

This work was supported by an Asklepios grant in 2014. Asklepios (http:// www.asklepiosgileadgrants.gr/) is a donation programme supported by Gilead Sciences, Greece, and proposals are reviewed by an independent committee. The funding source had no role in the design and conduct of the study; collection, management, analysis, and interpretation of the data; preparation, review, or approval of the manuscript; and decision to submit the manuscript for publication.

\section{Conflict of Interests}

None declared

\section{REFERENCES}

1. Beyrer C, Baral SD, van Griensven F, Goodreau SM, Chariyalertsak S, Wirtz AL, et al. Global epidemiology of HIV infection in men who have sex with men. Lancet. 2012;380(9839):367-77.

2. Beyrer C, Sullivan P, Sanchez J, Baral SD, Collins C, Wirtz AL, et al. The increase in global HIV epidemics in MSM. AIDS. 2013;27(17):2665-78.

3. Pharris A, Quinten C, Tavoschi L, Spiteri G, Amato-Gauci AJ. Trends in HIV surveillance data in the EU/EEA, 2005 to 2014: new HIV diagnoses still increasing in men who have sex with men. Euro Surveill. 2015;20(47). doi: 10.2807/1560-7917.ES.2015.20.47.30071.

4. Nikolopoulos GK, Sypsa V, Bonovas S, Paraskevis D, Malliori-Minerva M, Hatzakis A, et al. Big events in Greece and HIV infection among people who inject drugs. Subst Use Misuse. 2015;50(7):825-38. 
5. Paraskevis D, Paraschiv S, Sypsa V, Nikolopoulos G, Tsiara C, Magiorkinis G, et al. Enhanced HIV-1 surveillance using molecular epidemiology to study and monitor HIV-1 outbreaks among intravenous drug users (IDUs) in Athens and Bucharest. Infect Genet Evol. 2015;35:109-21.

6. European Centre for Disease Prevention and Control. Risk assessment on HIV in Greece. Stockholm: ECDC; 2012.

7. European Centre for Disease Prevention and Control. HIV testing: increasing uptake and effectiveness in the European Union. Evidence synthesis for Guidance on HIV testing. Stockholm: ECDC; 2010.

8. European Centre for Disease Prevention and Control. HIV testing: increasing uptake and effectiveness in the European Union. Stockholm: ECDC; 2010.

9. Pharris A, Spiteri G, Noori T, Amato-Gauci AJ. Ten years after Dublin: principal trends in HIV surveillance in the EU/EEA, 2004 to 2013. Euro Surveill. 2014 Nov 27;19(47). pii: 20968

10. von Elm E, Altman DG, Egger M, Pocock SJ, Gøtzsche PC, Vandenbroucke JP; STROBE Initiative. Strengthening the Reporting of Observational Studies in Epidemiology (STROBE) statement: guidelines for reporting observational studies. PLoS Med. 2007;4(10):e296. doi: 10.1371/journal.pmed.0040296.

11. Ferrer L, Loureiro E, Meulbroek M, Folch C, Perez F, Esteve A, et al. High HIV incidence among men who have sex with men attending a community-based voluntary counselling and testing service in Barcelona, Spain: results from the ITACA cohort. Sex Transm Infect. 2016;92(1):705 .

12. Meireles P, Lucas R, Carvalho C, Fuertes R, Brito J, Campos MJ, et al Incident risk factors as predictors of HIV seroconversion in the Lisbon cohort of men who have sex with men: first results, 2011-2014. Euro Surveill. 2015 Apr 9;20(14). pii: 21091.

13. Marcus U, Hickson F, Weatherburn P, Schmidt AJ. Prevalence of HIV among MSM in Europe: comparison of self-reported diagnoses from a large scale internet survey and existing national estimates. BMC Public Health. 2012 Nov 14;12:978. doi: 10.1186/1471-2458-12-978.

14. Molina JM, Capitant C, Spire B, Pialoux G, Cotte L, Charreau I, et al. On-demand preexposure prophylaxis in men at high risk for HIV-1 Infection. N Engl J Med. 2015 Dec 3;373(23):2237-46.

15. World Health Organization. Guidelines on when to start antiretroviral therapy and on pre-exposure prophylaxis for HIV. Geneva: WHO; 2015.

Received June 12, 2016 Accepted in revised form February 2, 2019 